\title{
Direct determination of molar absorption coefficients of several molecules in the lowest excited singlet states
}

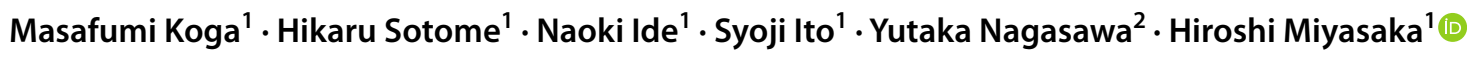

Received: 5 July 2021 / Accepted: 8 September 2021 / Published online: 21 September 2021

(c) The Author(s), under exclusive licence to European Photochemistry Association, European Society for Photobiology 2021

\begin{abstract}
Molar absorption coefficient of the lowest excited state is an indispensable information for the quantitative investigation of photochemical reactions by means of transient absorption spectroscopy. In the present work, we quantitatively estimated the molar absorption coefficients of the $\mathrm{S}_{1}$ state of the solute in three solution systems, Rhodamine B in ethanol, ZnTPP in DMF and $N, N$ '-bis(2,6-diisopropylphenyl)terrylene-3,4,11,12-tetracarboxydiimide (TDI) in chloroform, by perfectly bleaching the ground state molecules using the picosecond 532-nm laser pulse with a large number of photons. These solution systems were selected because no obvious photodegradation was detected in the present range of the excitation intensity. The molar absorption coefficient obtained by this method was verified by the numerical analysis of the excitation intensity dependence of the transient absorbance by taking into account the inner filter effect (absorption of the excitation light by the $\mathrm{S}_{1}$ state produced by the leading part of the pump pulse) and the decrease of the ground state molecules by the pump process (depletion). In addition, these molar absorption coefficients were confirmed by the comparison of relations between the excitation intensity and the transient absorbance of the $S_{1}$ state under the condition where the fraction of the excited solute is $\ll 10 \%$ by the femtosecond pulsed laser excitation. From these results, the error of the molar absorption coefficients was estimated to be $<5 \%$. These values can be used as reference ones for the estimation of molar absorption coefficients of other systems leading to the quantitative elucidation of the photochemical reactions detected by the transient absorption spectroscopy.
\end{abstract}

Keywords Transient absorption spectroscopy · Molar absorption coefficient of $S_{1}$ states · Ultrafast dynamics · Timeresolved detection

\section{Introduction}

Since the pioneering work of the flash photolysis by Norrish and Porter [1, 2], time-resolved detection of transient species has been utilized as a powerful tool for the elucidation of the mechanisms underlying various photochemical reactions and photoinduced phenomena. Among many detection methods, time-resolved measurement of electronic absorption spectra, which is known as transient absorption spectroscopy, has

Hiroshi Miyasaka

miyasaka@chem.es.osaka-u.ac.jp

1 Division of Frontier Materials Science and Center for Advanced Interdisciplinary Research, Graduate School of Engineering Science, Osaka University, Toyonaka, Osaka 560-8531, Japan

2 Department of Applied Chemistry, College of Life Sciences, Ritsumeikan University, 1-1-1 Noji-Higashi, Kusatsu, Shiga 525-8577, Japan been used as a principal tool. Because the frequency range of the electronic absorption is higher than those in other spectroscopic methods such as IR, ESR and NMR, higher temporal resolution can be obtained in the transient absorption spectroscopy. Moreover, electronic absorption spectra can cover, in principle, all the chemical species such as excited and ground states molecules, ions, ion radicals, neutral radicals, and so forth. We can assign the species appearing in the temporal evolution by comparing transient spectra with reference ones which were measured for the chemical species selectively prepared by appropriate methods. Analysis of the time dependence of the transient spectra reveals the reaction scheme of the photoinduced processes.

In addition to the above advantages, transient absorption spectroscopy can provide the quantitative information, such as reaction yields, of the transient species in the case where their absorption coefficients are known. For stable or quasi-stable radicals, chemical and/or electrochemical processes can selectively yield these radicals under 
steady-state conditions and molar absorption coefficients for these species can be determined with high accuracy. However, molar absorption coefficient of excited state molecules is generally difficult to correctly determine, owing to the short lifetime and difficulty in the precise evaluation of the volume of the exposure. In addition, inner filter effect, which is the absorption of the excitation light by the excited molecule during the pump pulse duration, also precludes the precise evaluation of the number of photons absorbed by the ground state molecules.

One of the simple and rigorous methods to overcome these difficulties is the perfect bleaching of the groundstate molecules in the exposed volume, which were applied to the determination of molar absorption coefficients of the long-living transient species such as triplet state [3]. Because this method employs the excitation light source containing a large number of photons such as flash lamps and giant nanosecond (ns) laser pulses, photodegradation and/or nonlinear optical process such as stepwise multiphoton absorption sometimes inhibit the accurate estimation of the absorption coefficient. Because the nonlinear optical processes are more easily induced with the shortening of the pulse duration, the perfect bleaching method becomes more difficult to apply to the determination of the transient species with the short lifetime.

The quantitative information on the population of $S_{1}$ state is, however, quite important to precisely elucidate photochemical reactions, because the lowest excited singlet state $\left(S_{1}\right.$ state) of molecules plays an important role as an initial state of almost all the photochemical reactions of polyatomic molecules owing to the rapid intramolecular internal conversion from higher excited states [4] and subsequent longitudinal vibrational relaxation in the condensed phase. In the present work, we have employed picosecond (ps) laser pulses at $532 \mathrm{~nm}$ to perfectly pump the solute molecule in solutions leading to the quantitative determination of the molar absorption coefficient of the $\mathrm{S}_{1}$ state of the solute. Among several systems to which we applied the excitation with rather high intensity pulse, we selected three systems that did not show remarkable photodegradation in the present excitation intensity range. By analyzing the excitation intensity dependence of transient absorbance and the concentration effect of the solute, we quantitatively evaluated the contribution of the nonlinear phenomena and obtained the absorption coefficient. In addition, we confirmed these values by comparing the absorption coefficients under the femtosecond pulsed laser excitation with much weaker intensities, which directly indicates that these molar absorption coefficients can be used as reference ones for the determination of the molar absorption coefficients of the $S_{1}$ state in other systems under the ultrafast laser spectroscopy.

\section{Experimental and analysis methods}

\subsection{Chemicals}

Zinc-tetraphenylporphyrin (ZnTPP, Sigma-Aldrich) was purified by flash column chromatography on alumina. A terrylenediimide, $N, N^{\prime}$-bis(2,6-diisopropylphenyl)terrylene3,4:11,12-tetracarboxydiimide (TDI, Yamada Chemical), Rhodamine-B ( RhB, Acros Organics), were used as received. Molecular structures of these materials are shown in Scheme 1. Dimethylformamide (DMF, Fujifilm-Wako, infinity-pure grade), ethanol (Fujifilm-Wako, infinity-pure grade) were used as solvents without further purification. The sample solution for the time resolved measurement was deaerated by irrigating with $\mathrm{N}_{2}$ gas. All the measurements were performed at $22 \pm 2{ }^{\circ} \mathrm{C}$.

\subsection{Apparatus}

Steady-state absorption spectra were recorded on a spectrophotometer (Hitachi U-3500). For the measurement under the perfect conversion of the solute molecules in the ground state to the excited state, a system of the transient absorption spectroscopy with a repetitive mode-locked $\mathrm{Nd}^{3+}$ :YAG laser was employed [5]. The SHG output $(532 \mathrm{~nm})$ with 15-ps fwhm was used as an excitation pulse. The diameter of the focal spot of the excitation beam is ca. $2 \mathrm{~mm}$, which was estimated by the introduction of the pin hole, measurements of the fluorescence spot size and the numerical analysis of the excitation intensity dependence of the transient absorbance as will be shown in later.

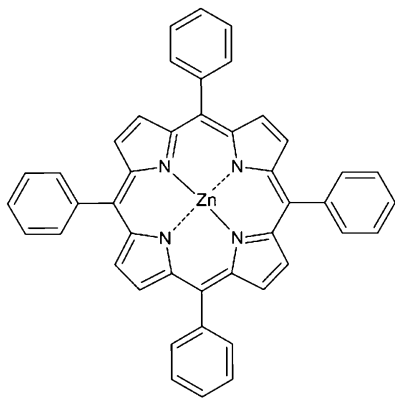

ZnTPP

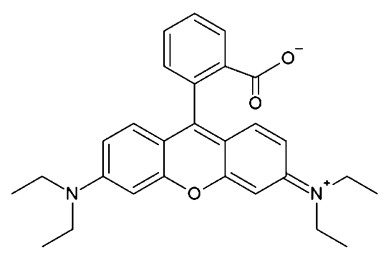

Rhodamine B

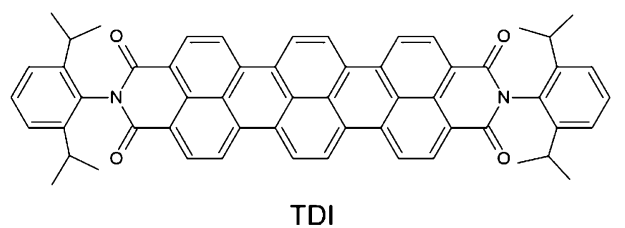

Scheme 1. Molecular structures used as solutes in the present study 
Remaining portion of the fundamental beam at $1064 \mathrm{~nm}$ was focused into a cell containing a $\mathrm{H}_{2} \mathrm{O} / \mathrm{D}_{2} \mathrm{O}$ mixture to convert to a white light continuum covering 400-980 nm as a probe pulse. This probe light was divided into two parts of signal and reference pulses. The signal pulse was focused at the spot of the excitation on the sample cell and the transmitted light was detected by a multichannel photodiode arrays (S4874, Hamamatsu) combined with a polychromator (Monospec 27, Jarrel Ash). The reference pulse was directly guided to the other photodiode arrays with another MCPD polychromator. Typical accumulation number for one transient absorption spectrum is $5-10$. The optical length of the cuvette was $2.0 \mathrm{~mm}$.

For the measurement of the transient absorption spectra by femtosecond laser pulses, a laser system with two noncollinear optical parametric amplifiers (NOPA, TOPASwhite, Light Conversion) pumped by a regeneratively amplified Ti:Sapphire laser (Solstice, Spectra-Physics) was used as a light source. The output of one of the NOPA systems was set at 532 or $550 \mathrm{~nm}$ and passed through a band-pass filter (Asahi Spectra) to narrow the spectral bandwidth less than $10 \mathrm{~nm}$ fwhm, which was used as an excitation beam. The diameter of the excitation spot at the sample position is ca. $100 \mu \mathrm{m}$, which was estimated by the introduction of the pin hole and the numerical analysis of the excitation intensity dependence of the transient absorbance as will be shown in later. The pulse width of the excitation pulse after the spectral narrowing is ca. $100 \mathrm{fs}$ fwhm. White-light continuum covering $400-930 \mathrm{~nm}$ was generated by focusing the output of another NOPA centered at $1100 \mathrm{~nm}$ into a rotating $\mathrm{CaF}_{2}$ crystal (thickness: $2 \mathrm{~mm}$ ), which was used as a probe pulse. The polarization of the pump pulse was set at the magic angle with respect to that of the probe pulse. The probe pulse was divided into signal and reference pulses. The signal pulse was guided into the sample cell and collimated to be overlapped with the pump pulse at the sample position. The transmitted signal beam was guided into the MCPD system with a polychromator (MSP1000-V, Unisoku). The reference pulse was directly guided into the other polychromator system. The bandwidth of transient absorption spectral measurements by both of ps and fs systems was estimated to be ca. $5 \mathrm{~nm}$, by comparing the spectra of HY-1 and V-10 filters with those measured by the steadystate spectrophotometer.

\subsection{Transient absorbance}

Transient absorbance is obtained as a difference between the spectra with and without the excitation. In the simple case where only the excited state, such as $S_{1}$ state, is populated after the photoexcitation, the transient absorbance is represented by Eq. (1).

$\Delta A=\left(\varepsilon_{e}-\varepsilon_{\mathrm{g}}\right) C_{g} \alpha L$

Here, $\varepsilon_{e}$ and $\varepsilon_{\mathrm{g}}$ are molar absorption coefficient of the excited state and that of the ground state respectively. $C_{g}, \alpha$, and $L$ are a fraction of molecules excited by the pump light, the concentration of the solute in the ground state, and the optical length of the cuvette. Because the lifetime of the excited state is finite, $\alpha$ is time-dependent. In addition, the optical axis of the probe light in the transient absorption spectroscopy in ultrafast time region is collinear or almost collinear with that of the pump pulse. Accordingly, the fraction of the excited molecules is also dependent on the distance from the surface of the sample. At the position, $x$, in the optical path of the sample $(0 \leq x \leq L)$, the decrease in the intensity of the probe light, $\mathrm{d} I_{\mathrm{m}}$, is given by Eq. (2).

$\mathrm{d} I_{\mathrm{m}}(x)=-I_{\mathrm{m}}(x)\left(\varepsilon_{e}-\varepsilon_{\mathrm{g}}\right) C_{g} a(x) \mathrm{d} x$.

Here, $a(x)$ is the fraction of the excited molecule at the position $x$. The value $\alpha$ in Eq. (1) is the integrated and averaged one along the optical length from $x=0$ to $L$. The transient absorbance is calculated by the intensity of the monitoring light passing through the sample. In the case where all of the ground state molecules are pumped up to the excited state, the transient absorbance is simply represented as $\Delta A=\left(\varepsilon_{e}-\varepsilon_{\mathrm{g}}\right) C_{g} L$ and we can obtain the molar absorption coefficient, $\varepsilon_{e}$ using $\varepsilon_{g}, C_{g}$ and $L$.

\subsection{Analysis of the excitation intensity dependence of the transient absorbance}

Excitation intensity dependence of the transient absorbance was analyzed using a numerical calculation method $[6,7]$. As illustrated in Scheme 2, this calculation takes into

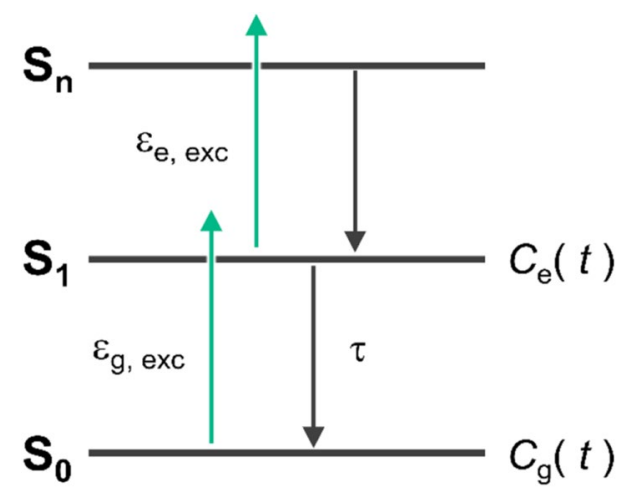

Scheme 2. Absorption processes of the excitation light by the ground state molecules and the excited state ones. The relaxation from higher excited state, $S_{n}$, to $S_{1}$ state, was assumed to take place instantaneously in the numerical analysis 
account the inner filter effect (absorption of the excitation light by the excited state), the decrease of the concentration of the ground state by the excitation and that in the excited state during the time duration of the excitation pulse along with the propagation of the pump pulse in the sample cell. Time and space steps for the numerical calculation were respectively $0.1 \mathrm{ps}$ and $10^{-3} \mathrm{~cm}$ for the 15 -ps laser pulse and $2 \mathrm{~mm}$ optical cuvette. For the femtosecond result, the time and space steps were $1 \mathrm{fs}$ and $10^{-3} \mathrm{~cm}$.

For the estimation of the focusing spot size of the excitation light, this numerical calculation was also applied. By the comparison of the experimental results with the calculated ones, the focusing spot size was determined on the assumption that the probe light monitors the uniform distribution of the excitation beam intensity in the spot area. The value thus obtained from the calculation is almost the same with that measured by the pin hole and/or the size of the fluorescence spot (within ca. $20 \%$ in a diameter).

\section{Results and discussion}

\subsection{Rhodamine B in ethanol solution}

Rhodamine $\mathrm{B}(\mathrm{RhB})$ has been used as an absorption and fluorescence standard in various solutions due to its excellent photodurability, high luminescence quantum yield, as well as large molar absorption coefficient in the ground state. RhB is under the equilibrium among several species as shown in Scheme 3 and this equilibrium is dependent on the solvent, $\mathrm{pH}$, temperature and so forth [8]. Judging from the $\mathrm{pH}$ of ethanol (ca. 9.4) and pKa of $\mathrm{RhB}$ (3.1) [9], RhB is in the zwitter ion form in ethanol solution.

Figure 1a shows the steady-state absorption spectrum of RhB in ethanol solution. The content of ethanol used in the present solution was $>99.8 \%$. Although we used several ethanol solvents with different content of ethanol such as $95 \%$, the absorption spectrum in these solutions did not show remarkable difference from Fig. 1a. In addition, even if we prepared the solution from Rhodamine B base or Rhodamine $\mathrm{B}$ hydrochloride, the absorption spectrum in the ethanol solution is almost the same. These results ensure that the same isomer, zwitter ion, is dominant in the ethanol solution at $22{ }^{\circ} \mathrm{C}$. The absorption peak at $543 \mathrm{~nm}$ in Fig. 1a can be
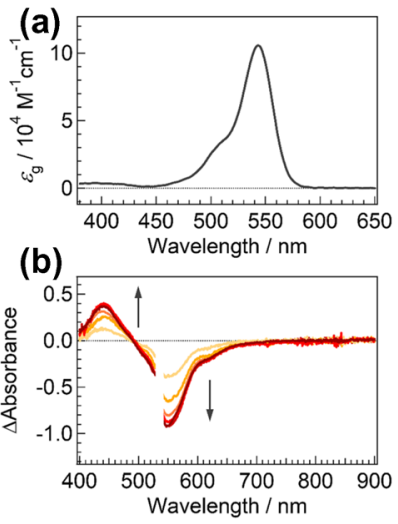

Fig. 1 a Steady-state absorption spectrum RhB in ethanol solution. b Transient absorption spectra of $\mathrm{RhB}$ with various excitation intensities of the ps $532 \mathrm{~nm}$ laser pulse, observed at 100 ps after the excitation of the solution with the concentration of $4.5 \times 10^{-5} \mathrm{M}$. The fluence of the laser pulse for each spectrum was $0.03,0.06,0.10,0.20$, 0.39 and $0.80 \mathrm{~mJ} /$ pulse. The focusing spot size was $2.0 \mathrm{~mm}^{2}$. The arrow in the figure indicates the increase in the fluence

assigned to $\mathrm{S}_{1} \leftarrow \mathrm{S}_{0}$ absorption band because this absorption band shows a mirror image of the fluorescence spectrum.

Figure $1 \mathrm{~b}$ shows transient absorption spectra of $\mathrm{RhB}$ measured at 100 ps after the excitation by the ps 532-nm laser pulse with various excitation intensities. The negative band around $545 \mathrm{~nm}$ and its tail around $600 \mathrm{~nm}$ are respectively assigned to the ground state bleaching and the stimulated emission. On the other hand, a positive signal around $438 \mathrm{~nm}$ is ascribable to the $\mathrm{S}_{\mathrm{n}} \leftarrow \mathrm{S}_{1}$ absorption band. In the present excitation condition, band shapes of the spectra are independent of the excitation intensity, indicating no remarkable production of the species other than the $S_{1}$ state in the present excitation intensity range.

Figure 2a shows the dependence of the transient absorbance at $438 \mathrm{~nm}$ and that at $543 \mathrm{~nm}$ on the excitation intensity for solutions with various concentrations of RhB. The absolute value of transient absorbance increases with increasing excitation intensity in rather low intensity range and it reaches the plateau value with further increase in the excitation intensity. These saturation features strongly suggest that $\mathrm{RhB}$ molecules in the ground state in the exposed volume are completely pumped to the excited state under the high excitation intensity.
Scheme 3. Equilibrium of Rhodamine B in the ground state
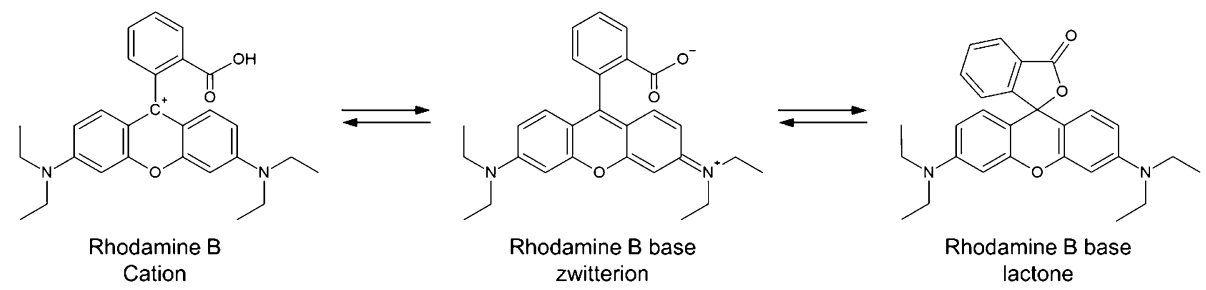

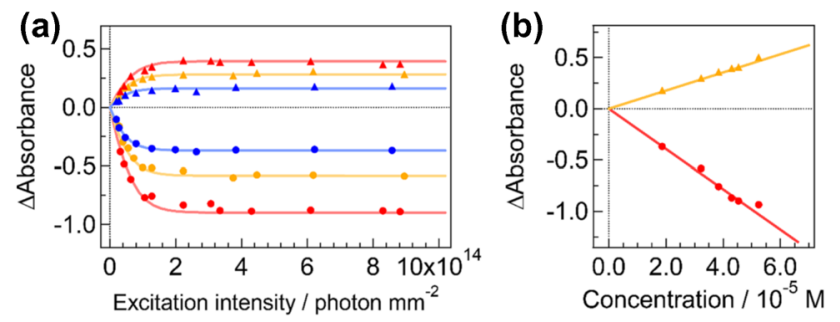

Fig. 2 a Excitation intensity dependencies of the transient absorbance at $438 \mathrm{~nm}$ (triangle) and that at $543 \mathrm{~nm}$ (circle) of $\mathrm{RhB}$ in ethanol solution. Concentration of RhB was $4.5 \times 10^{-5} \mathrm{M}$ (red), $3.3 \times 10^{-5} \mathrm{M}$ (yellow), and $1.9 \times 10^{-5} \mathrm{M}$ (blue), respectively. Solid lines are results of numerical simulations taking into account the inner filter effect and the bleaching of the ground state molecules (see text). b Relation between the transient absorbance at the plateau region and the concentration of the ground state; $543 \mathrm{~nm}$ (circles) and $438 \mathrm{~nm}$ (triangles)

In Fig. 2b, we show the relation between the transient absorbance at the plateau region and the concentration of $\mathrm{RhB}$ in the ground state, exhibiting the linear relation with the zero intercept. This result supports the perfect bleaching of the ground state at the region where the transient absorbance shows the plateau value. From this linear relation, the difference of molar absorption coefficient, $\varepsilon_{e}-\varepsilon_{\mathrm{g}}$, at $438 \mathrm{~nm}$ was obtained to be $46,700 \mathrm{M}^{-1} \mathrm{~cm}^{-1}$. Using $\varepsilon_{\mathrm{g}}$ of $2100 \mathrm{M}^{-1} \mathrm{~cm}^{-1}$ at $438 \mathrm{~nm}, \varepsilon_{e}$ at $438 \mathrm{~nm}$ was determined to be $48,800 \mathrm{M}^{-1} \mathrm{~cm}^{-1}$. Transient absorption spectrum with the molar absorption coefficient, $\varepsilon_{e}-\varepsilon_{\mathrm{g}}$, as ordinate is shown in Fig. 3. This spectrum corresponds to the difference absorption spectrum between the $S_{1}$ and $S_{0}$ states including the contribution from the stimulated emission. Because the transient absorption spectra were measured at $100 \mathrm{ps}$ after the excitation, the slight decrease due to the decay of the $S_{1}$ state was corrected using the fluorescence lifetime.

To confirm the validity of these values, the excitation intensity dependence of the transient absorbance was numerically calculated using the molar absorption coefficients of the $S_{1}$ state and that of the ground state at the excitation wavelength, $532 \mathrm{~nm}$. Because the value of the molar absorption coefficient of the $S_{1}$ state at $532 \mathrm{~nm}$ was not directly determined owing to the introduction of the notch filter to

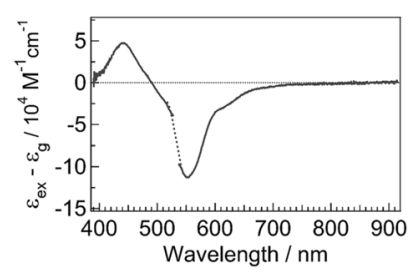

Fig. 3 Difference absorption spectrum between the $S_{1}$ and $S_{0}$ states of $\mathrm{RhB}$ in ethanol solution. The ordinate is given as $\left(\varepsilon_{e}-\varepsilon_{\mathrm{g}}\right)$. The spectrum around $532 \mathrm{~nm}$ was interpolated by the femtosecond result in Fig. 12 avoid the scattering of the excitation light at $532 \mathrm{~nm}$, the spectrum obtained by the femtosecond laser pulsed excitation (Fig. 12) was used for the determination of the molar absorption coefficient at $532 \mathrm{~nm}$. In this numerical calculation, the bleaching of the ground state molecule and the inner filter effect were taken into account as was shown in Scheme 2. Solid lines in Fig. 2a, which were thus calculated, well reproduce the excitation intensity dependencies of the transient absorbance at 438 and $543 \mathrm{~nm}$, ensuring the absorption coefficient determined by the present procedure.

\subsection{ZnTPP in DMF solution}

ZnTPP also serves as one of standard samples in various photochemical systems including transient absorption spectroscopy. Figure 4a shows a steady-state absorption spectrum of ZnTPP in DMF solution. The absorption signal around 470-620 $\mathrm{nm}$ is assigned to the Q band and the strong band in the shorter wavelength region can be ascribable to the Soret band [9]. Figure 4b shows transient absorption spectra at $100 \mathrm{ps}$ after the excitation with various intensities of the 532-nm laser pulse. Positive absorption with a peak at $454 \mathrm{~nm}$ is observed with dip signals at 558, 600 and $665 \mathrm{~nm}$. The dip signal at $558 \mathrm{~nm}$ is ascribable to the ground state bleaching. In the signal around $600 \mathrm{~nm}$, contribution of the stimulated emission is included in addition to the ground state bleaching. On the other hand, the dip signal around $665 \mathrm{~nm}$ is attributed to the stimulated emission. The spectrum with these signals is safely ascribable to the $\mathrm{S}_{\mathrm{n}} \leftarrow \mathrm{S}_{1}$ absorption of ZnTPP. Actually, the decay time constant of these signals agrees with the fluorescence lifetime (1.9 ns) and the spectrum
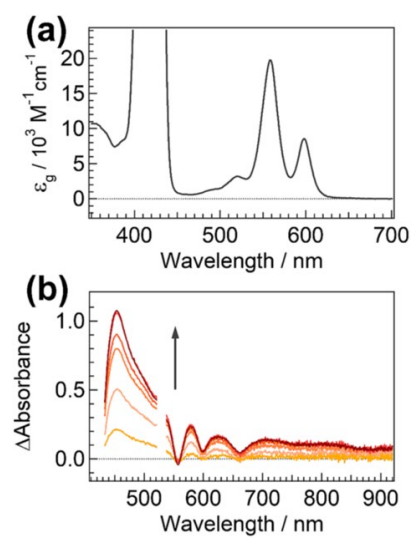

Fig. 4 a Steady-state absorption spectrum of ZnTPP in DMF. b Transient absorption spectra of ZnTPP in DMF observed at $100 \mathrm{ps}$ with various excitation intensities. The concentration of ZnTPP was $4.0 \times 10^{-4} \mathrm{M}$. The fluence of the laser pulse for each spectrum was $0.2,0.8,1.4,2.0,2.7$ and $3.4 \mathrm{~mJ} /$ pulse. The focusing spot size was $2.50 \mathrm{~mm}^{2}$. The arrow in the figure indicates the increase in the fluence 
of the $S_{1}$ state is replaced with the $T_{n} \leftarrow T_{1}$ absorption with the time constant of the fluorescence lifetime [9]. Figure $4 \mathrm{~b}$ also shows the increase of the transient absorbance with an increase in the excitation intensity in the region with rather low excitation intensity and saturation tendency was observed with further increase in the intensity. The spectral band shape was, however, independent of the excitation intensity in the present range, indicating no remarkable production of the species other than the $\mathrm{S}_{1}$ state.

Figure $5 \mathrm{a}$ shows the excitation intensity dependence of $\Delta A$ at $454 \mathrm{~nm}$ of three solutions with different concentrations of ZnTPP. As was shown in Fig. 4b, the transient absorbance at $454 \mathrm{~nm}$ monotonously increases with increasing excitation intensity and reaches the plateau value. With an increase in the concentration of ZnTPP in the ground state, this plateau value linearly increases with an intercept of zero as shown in Fig. 5b. This result ensures that the plateau value is due to the perfect bleaching of the ground state absorption. From the slope of Fig. 5b, the difference molar absorption coefficient, $\varepsilon_{e}-\varepsilon_{\mathrm{g}}$, at $454 \mathrm{~nm}$ was obtained to be $44,000 \mathrm{M}^{-1} \mathrm{~cm}^{-1}$. Using $\varepsilon_{e}$ at $454 \mathrm{~nm}$, the $\varepsilon_{e}$ value was determined to be $44,800 \mathrm{M}^{-1} \mathrm{~cm}^{-1}$. The difference absorption spectrum between the $S_{1}$ and $S_{0}$ states including the stimulated emission of ZnTPP in DMF is shown in Fig. 6 with the ordinate of the molar absorption. Owing to the strong absorption of the Soret band, the spectrum in the wavelength region $<440 \mathrm{~nm}$ is less reliable. As performed for $\mathrm{RhB}$, the slight decrease due to the decay of the $S_{1}$ state was corrected using the fluorescence lifetime of $1.9 \mathrm{~ns}$.

To confirm these values, the relation between the excitation intensity and transient absorbance was calculated as for Fig. 2a. Solid lines in Fig. 5a, which were calculated as for Fig. 2a, reproduce the excitation intensity dependencies of the transient absorbance at $454 \mathrm{~nm}$, ensuring the absorption coefficient determined by the present procedure.
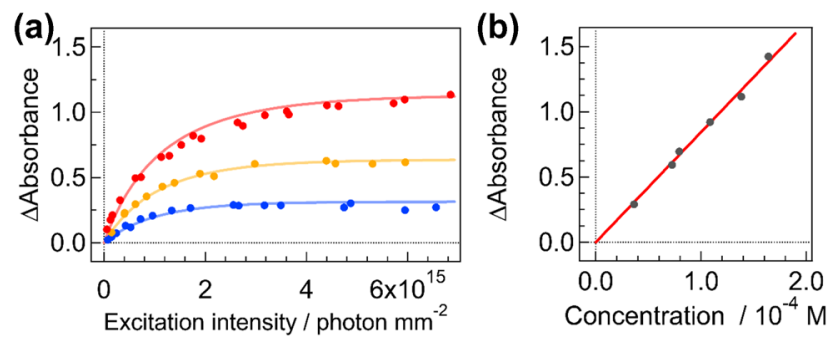

Fig. 5 a Transient absorbance at $454 \mathrm{~nm}$ as a function of the excitation intensity for three different concentrations of ZnTPP in DMF solution (red: $1.4 \times 10^{-4} \mathrm{M}$, yellow: $7.3 \times 10^{-5} \mathrm{M}$, blue: $3.6 \times 10^{-5} \mathrm{M}$ ). d Relation between the transient absorbance at $454 \mathrm{~nm}$ at the plateau region and the concentration of ZnTPP. The optical length of the cuvette was $2.0 \mathrm{~mm}$

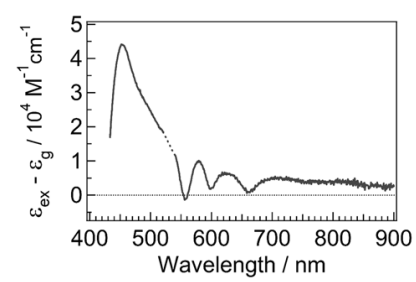

Fig. 6 Difference absorption spectrum between the $S_{1}$ and $S_{0}$ states of ZnTPP in DMF solution. The ordinate is given as $\varepsilon_{e}-\varepsilon_{\mathrm{g}}$. The spectrum around $532 \mathrm{~nm}$ was interpolated by the cubic spline curve

\subsection{TDI in chloroform solution}

Terrylenediimide derivatives have large absorption coefficients in the ground state and high photo-durability [10]. Steady-state absorption spectrum of TDI in chloroform solution is shown in Fig. 7a, where the $\mathrm{S}_{1} \leftarrow \mathrm{S}_{0}$ absorption appears with vibrational progression in the wavelength region of 500-680 nm [11].

Transient absorption spectra obtained with various excitation intensities are shown in Fig. 7b. Signals due to the ground-state bleaching and stimulated emission comprise a mirror-image with the center wavelength around $660 \mathrm{~nm}$. A positive absorption peak at $870 \mathrm{~nm}$ can be ascribable to the $\mathrm{S}_{\mathrm{n}} \leftarrow \mathrm{S}_{1}$ absorption band because the decay time constant of this band is the same with the fluorescence lifetime. Transient absorbance monotonously increases with an increase in the excitation intensity in the low range of the laser power and the saturation tendency was observed with further increase in the fluence. The spectral band shape was, however, independent of the excitation intensity in the
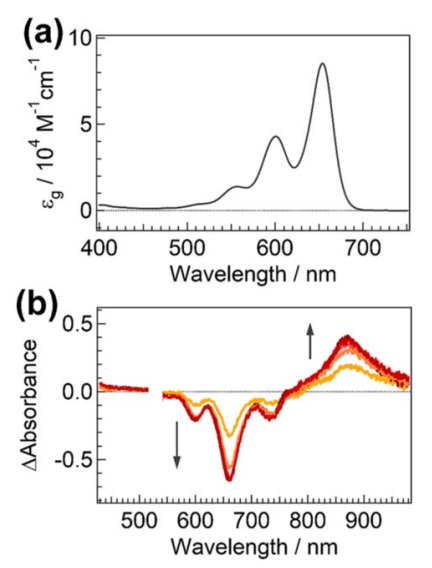

Fig. 7 a Steady-state absorption spectrum of TDI in $\mathrm{CHCl}_{3}$. b Transient absorption spectra of TDI obtained with different excitation intensity. Transient spectra were measured at 100 ps after the excitation of the solution with the concentration of $6.1 \times 10^{-5} \mathrm{M}$. The fluence of the laser pulse for each spectrum was 0.2, 0.7, 1.0, 1.6 and $2.3 \mathrm{~mJ} / \mathrm{pulse}$. The focusing spot size was $2.50 \mathrm{~mm}^{2}$. The arrow in the figure indicates the increase in the fluence 

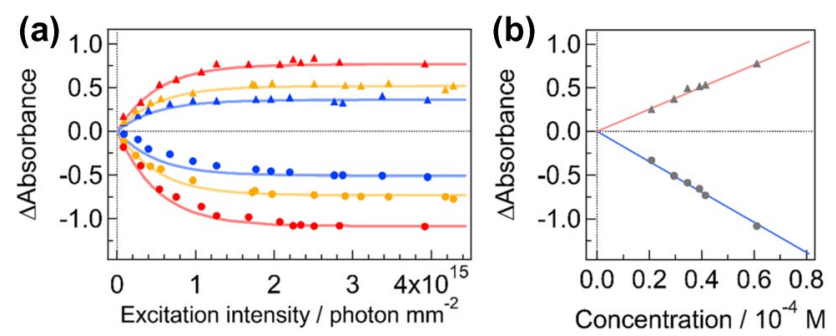

Fig. 8 a Transient absorbance at $870 \mathrm{~nm}$ (triangles) and $654 \mathrm{~nm}$ (circles) as a function of the excitation intensity for three different concentrations of TDI in chloroform solution (red: $6.1 \times 10^{-5} \mathrm{M}$, yellow: $4.1 \times 10^{-5} \mathrm{M}$, blue: $\left.2.9 \times 10^{-5} \mathrm{M}\right)$. b Relation between the transient absorbance at $870 \mathrm{~nm}$ (triangles) and $654 \mathrm{~nm}$ (circles) at the plateau region and the concentration of TDI in the ground state. The optical length of the cuvette was $2.0 \mathrm{~mm}$

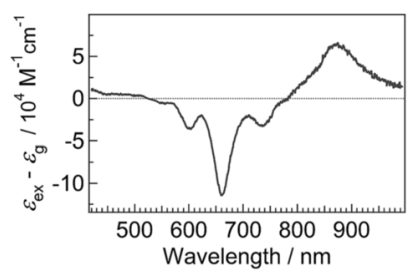

Fig. 9 Difference absorption spectrum between the $S_{1}$ and $S_{0}$ states of TDI in chloroform solution. The ordinate is given as $\varepsilon_{e}-\varepsilon_{\mathrm{g}}$. The spectrum around $532 \mathrm{~nm}$ was interpolated by the cubic spline curve

present range, indicating no remarkable production of the species other than the $S_{1}$ state.

Figure $8 \mathrm{a}$ shows the excitation intensity dependencies of the transient absorbance at 654 and $870 \mathrm{~nm}$. Both the absolute values of the negative signal at $654 \mathrm{~nm}$ and the positive one at $870 \mathrm{~nm}$ monotonously increase with increasing excitation intensity and reach the plateau value. With an increase in the concentration in the ground state, these plateau values linearly increase with an intercept of zero as shown in Fig. 8b, ensuring that the plateau value is due to the perfect bleaching of the ground state absorption. Because TDI has no absorption at $870 \mathrm{~nm}$ in the ground state, the molar absorption coefficient, $\varepsilon_{e}$, at $870 \mathrm{~nm}$ was determined to be $64,100 \mathrm{M}^{-1} \mathrm{~cm}^{-1}$ from the slope of Fig. $8 \mathrm{~b}$. The difference absorption spectrum between the $S_{1}$ and $S_{0}$ states of TDI including the stimulated emission in chloroform solution is shown in Fig. 9 with the ordinate of the molar absorption coefficient. As performed for RhB and ZnTPP, the slight decrease due to the decay of the $S_{1}$ state was corrected using the fluorescence lifetime.

To confirm the molar absorption coefficient, the relation between the excitation intensity and transient absorbance was calculated as for Figs. 2a and 5a. Solid lines in Fig. 8a, which were thus calculated, reproduce the excitation intensity dependence of the transient absorbance at $870 \mathrm{~nm}$, ensuring the absorption coefficient determined by the present procedure.

\subsection{Relative determination of the molar absorption coefficients of the $S_{1}$ state using femtosecond pulsed laser}

In the femtosecond (fs) transient absorption spectroscopy, the number of photons in the excitation pulse is much smaller than that in the ps one. Hence, the fraction of the molecule excited by the pump pulse is usually very low and the linear relation between the transient absorbance and the excitation intensity could be expected in most of the case where the molar absorption coefficient of the transient species at the excitation wavelength is not much larger than that in the ground state. Accordingly, we can estimate the molar absorption coefficient by comparing the relation between the transient absorbance and the excitation intensity of the sample with that of the reference one for which the molar absorption coefficient is already known.

As indicated in Eq. (1), the transient absorbance of the reference and target molecule monitored at the wavelength $\lambda_{1}$ and $\lambda_{2}$ are respectively represented by Eqs. (3) and (4).

$\Delta A_{R}^{\lambda_{1}}=\left(\varepsilon_{e, R}^{\lambda_{1}}-\varepsilon_{g, R}^{\lambda_{1}}\right) \alpha c_{R} L$,

$\Delta A_{T}^{\lambda_{2}}=\left(\varepsilon_{e, T}^{\lambda_{2}}-\varepsilon_{g, T}^{\lambda_{2}}\right) \alpha^{\prime} c_{T} L$.

Here, $\varepsilon_{e, R}^{\lambda_{1}}$ and $\varepsilon_{e, T}^{\lambda_{2}}$ are respectively the molar absorption coefficient of the excited states of the reference system and that of the target sample. As mentioned for Eq. (1), $\alpha$ and $\alpha^{\prime}$ are the fractions of the excited molecule and time dependent. The optical length of the cuvette is given as $L$. In the case where the inner filter effect is ignored and the fraction of the excited state is small (<a few \%), $\alpha$ and $\alpha^{\prime}$ can be described simply by the number of incident photons, $N_{p h}$, the exposed volume, $V$, and the absorbance of the ground state, $A$, at the excitation wavelength, $\lambda_{p}$.

$\alpha=\frac{c_{e, R}}{c_{R}}=\frac{1}{c_{R}} \frac{N_{p h}}{V N_{A}}\left(1-10^{-A_{R}^{\lambda_{p}}}\right)$,

$\alpha^{\prime}=\frac{c_{e, T}}{c_{T}}=\frac{1}{c_{T}} \frac{N_{p h}}{V N_{A}}\left(1-10^{-A_{T}^{\lambda_{p}}}\right)$.

The ratio of $\alpha$ and $\alpha^{\prime}$ is given by Eq. (7)

$\frac{\alpha}{\alpha^{\prime}}=\frac{c_{T}}{c_{R}} \cdot \frac{1-10^{-A_{R}^{\lambda_{p}}}}{1-10^{-A_{T}^{\lambda_{p}}}}$.

The transient absorbance is represented by Eq. (8). 

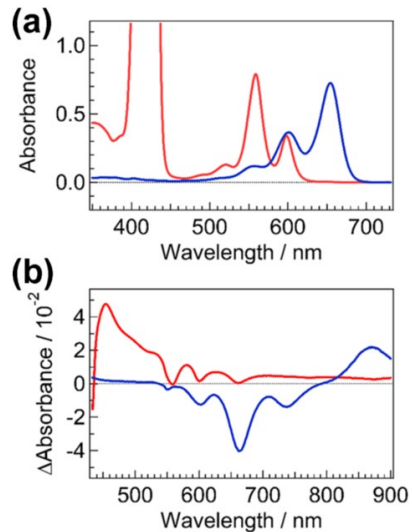

Fig. 10 a Steady-state absorption spectra of TDI in chloroform (blue) and ZnTPP in DMF (red). b Transient absorption spectra of TDI (blue) and ZnTPP (red) 100 ps after the excitation with a fs laser pulse at $550 \mathrm{~nm}(18 \mathrm{~nJ} /$ pulse $)$

$\frac{\Delta A_{T}^{\lambda_{2}}}{\Delta A_{R}^{\lambda_{1}}}=\frac{\left(\varepsilon_{e, T}^{\lambda_{2}}-\varepsilon_{g, T}^{\lambda_{2}}\right)}{\left(\varepsilon_{e, R}^{\lambda_{1}}-\varepsilon_{g, R}^{\lambda_{1}}\right)} \frac{\alpha^{\prime} c_{T}}{\alpha c_{R}}$

In the actual measurement, $\alpha$ and $\alpha^{\prime}$ are dependent on the delay time after the excitation, because the lifetime of the excited state is finite. Figure 10a shows steady-state absorption spectra of ZnTPP in DMF and TDI in chloroform solutions used for the transient absorption spectra. Figure 10b exhibit the transient absorption spectra of these systems observed at $100 \mathrm{ps}$ following the excitation with the 550-nm fs laser pulse. Band shapes of these spectra are in agreement with those observed by the ps 532-nm excitation in Figs. 4 and 7 . The relation between the transient absorbance and the excitation intensity is shown in Fig. 11, where the signal of TDI at $870 \mathrm{~nm}$ and that of ZnTPP at $454 \mathrm{~nm}$ are plotted. As this figure shows, linear relationships between the transient absorbance and the excitation intensity hold in the excitation intensity range up to ca. $18 \mathrm{~nJ}$. Dotted lines in the figure are results calculated using Eqs. (4-7) with the molar absorption coefficients of the $S_{1}$ state determined by the ps experiments. These curves well reproduce the experimental results in the excitation range $\leq 18 \mathrm{~nJ}$. On the other hand, solid lines in the figure are the results of the calculation taking into account the decrease of the ground state molecules by the pump process and the inner-filter effect as performed for the analyses of the ps results. Molar absorption coefficients determined by the ps experiments were used in this calculation. Although the slight decrease of the transient absorbance was observed in the excitation intensity higher than ca. $20 \mathrm{~nJ}$, the difference from the dotted lines is small, indicating the small fraction of the excited molecules. Actually, from the molar absorption coefficients of the $S_{1}$ state

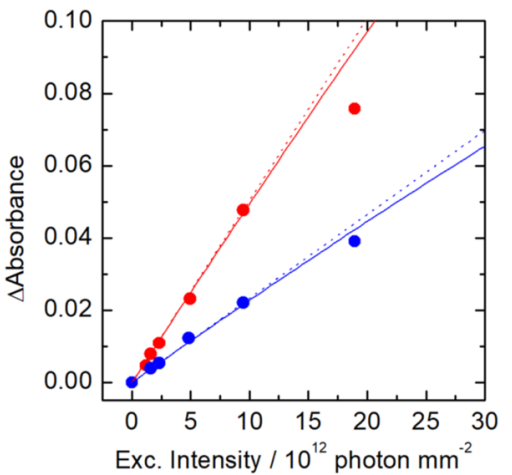

Fig. 11 Relation between the $\triangle$ Absorbance of ZnTPP monitored at $454 \mathrm{~nm}$ (red) and that of TDI at $870 \mathrm{~nm}$ (blue) and the 550-nm excitation intensity. The monitoring time is 100 ps after the excitation. Dotted lines in the figure are results calculated using Eqs.(4-7) with the molar absorption coefficients of the $S_{1}$ state determined by the ps experiments. Solid lines are the results of the calculation taking into account the decrease of the ground state molecules by the pump process and the inner filter effect as performed for the analyses of the ps results

and the concentrations of the ground state, the fraction of the $S_{1}$ state for the TDI system is estimated to be $3.9 \%$ and that for the ZnTPP system is $2.9 \%$ at the excitation intensity of $18 \mathrm{~nJ}$.

We also applied simple analysis with the linear least square method. In this analysis, the experimental result in the excitation $\leq 18 \mathrm{~nJ}$ was used. The molar absorption coefficient, $\varepsilon_{\mathrm{e}}$, of TDI at $870 \mathrm{~nm}$ was estimated to be $65,100 \mathrm{M}^{-1} \mathrm{~cm}^{-1}$ using $\varepsilon_{\mathrm{e}}$ and $\varepsilon_{\mathrm{g}}$ of ZnTPP at $454 \mathrm{~nm}$ as a reference. The $\varepsilon_{\mathrm{e}}$ value thus estimated was almost the same with that directly determined by the picosecond laser experiment, $64,100 \mathrm{M}^{-1} \mathrm{~cm}^{-1}$. This result indicates that the present method in the fs experiment can relatively determine the molar absorption coefficient of $S_{1}$ states.

To verify the applicability of this measurement using ZnTPP as the reference data, we also used RhB as a target sample. Figure 12a shows absorption spectra of ZnTPP and $\mathrm{RhB}$ used in this experiment. For the femtosecond measurement, we used 532-nm laser pulse as an excitation light, because $\mathrm{RhB}$ in ethanol exhibits fluorescence at $550 \mathrm{~nm}$ and the excited-state population is strongly influenced by the stimulated emission induced by the excitation pulse at $550 \mathrm{~nm}$.

Figure $12 \mathrm{~b}$ and $\mathrm{c}$ show the transient absorption spectra of ZnTPP and RhB excited with a fs 532-nm laser pulse with an excitation energy of $18 \mathrm{~nJ}$. Band shapes of these spectra are in agreement with those obtained by the ps 532-nm laser pulse.

The relation between the transient absorbance and the excitation intensity of the fs $532-\mathrm{nm}$ laser pulses is shown in Fig. 13, where the signal of RhB at $438 \mathrm{~nm}$ (green) and 


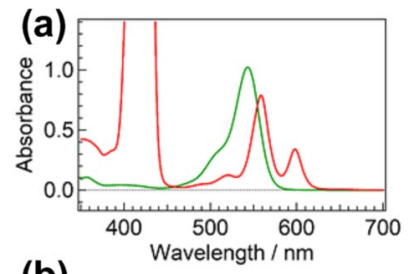

(b)

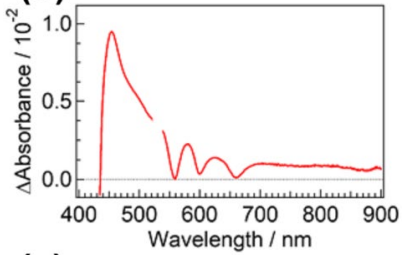

(c)

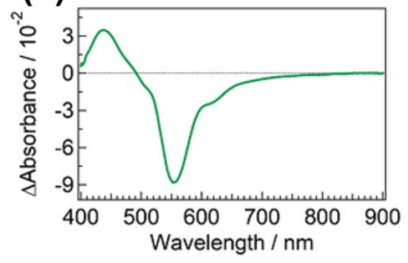

Fig. 12 a Steady-state absorption spectra of ZnTPP in DMF (red) and $\mathrm{RhB}$ in ethanol (Green). (b,c) Transient absorption spectra of ZnTPP (b) and $\mathrm{RhB}(\mathbf{c})$ excited with 532-nm, $18 \mathrm{~nJ}$ laser pulse

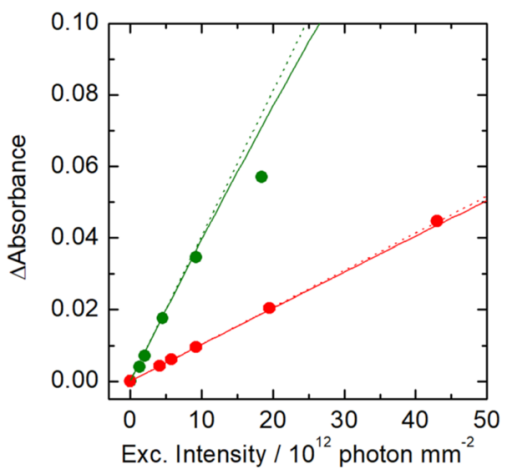

Fig. 13 Relation between the $\triangle$ Absorbance of ZnTPP monitored at $454 \mathrm{~nm}$ (red) and that of RhB at $438 \mathrm{~nm}$ (green) and the 530-nm excitation intensity. The monitoring time is $100 \mathrm{ps}$ after the excitation. Dotted lines in the figure are results calculated using Eqs.(4-7) with the molar absorption coefficients of the $S_{1}$ state determined by the ps experiments. Solid lines are the results of the calculation taking into account the decrease of the ground state molecules by the pump process and the inner filter effect as performed for the analyses of the ps results

that of ZnTPP at $454 \mathrm{~nm}$ (red) are plotted. The linear relations are obtained in both of the samples in the region $<$ ca. $20 \mathrm{~nJ}$. Dotted lines in the figure are results calculated using Eqs. (4-7) with the molar absorption coefficients of the $S_{1}$ state determined by the ps experiments. These curves well reproduce the experimental results, at least, in the excitation range $\leq 18 \mathrm{~nJ}$. On the other hand, solid lines in the figure are the results of the calculation taking into account the decrease of the ground state molecules by the pump process and the inner-filter effect as performed for the analyses of the ps results. Molar absorption coefficients which were determined by the calculated curves were used in this calculation. Although the slight decrease of the transient absorbance in the calculation curve was observed in the excitation intensity higher than ca. $20 \mathrm{~nJ}$, the difference from the dotted lines is small, indicating the small fraction of the excited molecules. Actually, from the molar absorption coefficients of the $S_{1}$ state and the concentrations of the ground state, the fraction of the $S_{1}$ state for the RhB system is estimated to be $7.7 \%$ and that for the ZnTPP system is $0.6 \%$ at the excitation intensity of $18 \mathrm{~nJ}$.

We also applied simple analysis with the linear least square method. In this analysis, we used the experimental result of the ZnTPP system as the reference sample. The experimental results in the excitation range $\leq 18 \mathrm{~nJ}$ was used. The molar absorption coefficient, $\varepsilon_{\mathrm{e}}-\varepsilon_{\mathrm{g}}$, of $\mathrm{RhB}$ at $438 \mathrm{~nm}$ was obtained to $45,500 \mathrm{M}^{-1} \mathrm{~cm}^{-1}$ using $\varepsilon_{\mathrm{e}}$ and $\varepsilon_{\mathrm{g}}$ of ZnTPP at $454 \mathrm{~nm}$ as a reference. The $\varepsilon_{\mathrm{e}}$ value obtained by the fs measurements almost the same with that directly determined by the picosecond laser experiment, $46,700 \mathrm{M}^{-1} \mathrm{~cm}^{-1}$. This result indicates that the present method in the fs experiment can relatively determine the molar absorption coefficient. From these results, it is concluded that the values relatively determined is within an error of less than at most $5 \%$.

\section{Summary}

We have determined molar absorption coefficients of the $\mathrm{S}_{1}$ state of three systems by the complete bleaching of the ground state molecule using a picosecond pulsed laser. As written in the introductory part, no obvious photodegradation was confirmed for the three systems in the present range of the excitation light intensity. However, with a further increase in the excitation light intensity, the decrease of the transient absorbance from the plateau values was observed, which is probably due to the photodegradation by the repeated photoexcitation of the $S_{1}$ state.

Generally, not only the $S_{0}$ state but also the $S_{1}$ state has absorption bands at the excitation wavelength. Accordingly, the light absorption of the ground state molecules occurs in competition with the absorption by the $\mathrm{S}_{1}$ state during the pulse duration. This second photon absorption process is the origin of the inner filter effect, which suppresses the increase of the production of the $S_{1}$ state with an increase in the excitation intensity. The second photon absorption pumps $\mathrm{S}_{1}$ state molecule up to higher electronic states. Although the lifetime of higher excited states is typically very short ( $\ll 1 \mathrm{ps}$ ) and the rapid relaxation yields the $S_{1}$ state again in most of the case, it is important for the suppression of the 
photodegradation of the sample that the yield of photoreaction from higher excited state is very small. In the samples shown here, no obvious photodegradation was confirmed in the present range of the excitation light intensity, indicating that the reaction yield in higher excited states is very small.

It is also worth noting that the typical reaction in higher excited states is the photoionization. In the present systems, photoionization via the stepwise two-photon absorption was not observed. This is because the excitation light was in the visible region and the level of the higher excited state attained by the second photon absorption is lower than the ionization threshold of these solutes in solutions. In the case where UV light is used for the excitation, however, we should precisely check the contribution of the two-photon ionization [12-14]. Therefore, the complete bleaching method using a picosecond laser needs several conditions to avoid degradation and ionization through the stepwise two-photon absorption processes.

On the other hand, the number of photons in the femtosecond laser pulse used for transient absorption measurement is much smaller and the fraction of the molecules excited by the pulsed laser is $<$ ca. $10 \%$. In this case, the transient absorbance is linearly in proportion with the excitation intensity. In this excitation intensity range, the molecular absorption coefficient of the system, in principle, can be relatively determined using the reference system in the condition where some nonlinear processes such as off-resonant two-photon absorption of the solvent can be avoided. This method is applicable to the system whose lifetime of the excited singlet state is too short to completely pump the ground-state molecules by the picosecond pulsed excitation. The comparison of the excitation intensity dependence of the target system with that of the reference system under the same condition can relatively provide the molar absorption coefficient in the excitation intensity range where the linear relation between the transient absorbance and the pump intensity holds, as shown in Figs. 11 and 13.

The numerical simulation for the relation between the transient absorbance and the excitation intensity is also effective to quantitatively estimate the molar absorption coefficient of the $S_{1}$ state. By taking into account the molar absorption coefficient of the $S_{1}$ state at the excitation wavelength leading the second photon absorption and the decrease of the number of the ground state molecules, the excitation intensity dependence could be simulated and can be compared with the experimental result.

The molar absorption coefficient used in this numerical simulation was, however, the values obtained at $100 \mathrm{ps}$. During the excitation of the ps laser pulse (fwhm $15 \mathrm{ps),} \mathrm{all} \mathrm{the}$ excited state is not fully relaxed to the $S_{1}$ state under the thermal equilibrium with the solvent and some portion is under the vibrational cooling. Hence, the molar absorption coefficient at the pump wavelength during the pulse duration is not the same with that at 100 ps. This difference may lead to the slight deviation of the simulated results from the experimental results. For the femtosecond experiments, this difference may cause large deviation because the pulse duration (fwhm $100 \mathrm{fs}$ ) is comparable with or shorter than time constants of the conversion of the excited electronic states, the intramolecular vibrational redistribution, and the lifetime of higher excited states. These effects may induce the difference between the experimental and simulated results, as observed in Figs. 11 and 13. In the present systems, however, the linear relation could be obtained in the region where the transient absorbance is smaller than ca. 0.05. Although this value is a practical one, it is possible to estimate the molar absorption coefficient by carefully confirming the linear relation between the transient absorbance and the excitation intensity.

Supplementary Information The online version contains supplementary material available at https://doi.org/10.1007/s43630-021-00104-z.

Acknowledgements The authors appreciate Mr. Yuto Masaoka and Mr. Tsubasa Hidaka at Ritsumeilkan University for their assistance in the fs experiments.

Funding This work was supported partly by JSPS KAKENHI Grant Numbers JP26107002 and 21H01889 to HM, No. 19J13917 for JSPS Research Fellow to M.K and the Research Foundation for Opto-Science and Technology to HS.

\section{Declarations}

Conflict of interest There are no conflicts to declare.

\section{References}

1. Norrish, R. G. W., \& Porter, G. (1949). Chemical reactions produced by very high light intensities. Nature, 164, 658. https://doi. org/10.1038/164658a0

2. Porter, G. (1950). Flash photolysis and spectroscopy. A new method for the study of free radical reactions. Proceedings of the Royal Society of London A, 200, 284-300. https://doi.org/10.1098/ rspa.1950.0018

3. Carmichael, I., \& Hug, G. L. (1983). Triplet-triplet absorption spectra of organic molecules in condensed phase. Journal of Physical and Chemical Reference Data, 15, 1-250. https://doi. org/10.1063/1.555770

4. Kasha, M. (1950). Characterization of electronic transitions in complex molecules. Discussions of the Faraday Society, 9, 14-19. https://doi.org/10.1039/DF9500900014

5. Miyasaka, H., Moriyama, T., \& Itaya, A. (1996). Direct detection of the hole migration along the polymer chain: Poly(N-vinylcarbazole) in 1,2-dichloroethane solution as revealed by picosecond transient absorption spectroscopy and dichroism measurements. Journal of Physical Chemistry, 100, 12609-12615. https://doi. org/10.1021/jp960549d

6. Miyasaka, H., Masuhara, H., \& Mataga, N. (1983). Picosecond absorption spectra and relaxation processes of the excited singlet 
state of pyrene in solution. Laser Chemistry, 1, 357-386. https:// doi.org/10.1155/LC.1.357

7. Murakami, M., Miyasaka, H., Okada, T., Kobatake, S., \& Irie, M. (2004). Dynamics and mechanisms of multiphoton gated photochromic reaction of diarylethene derivative. Journal of the American Chemical Society, 126, 14764-14772. https://doi.org/ 10.1021/ja049177+

8. Helttunen, K., Prus, P., Luostarinen, M., \& Nissinen, M. (2009). Interaction of aminomethylated resorcinarenes with rhodamine B. New Journal of Chemistry, 33, 1148-1154. https://doi.org/10. 1039/B820409H

9. Arbeloa, I. L., \& Ojeda, P. R. (1981). Molecular forms of rhodamine B. Chemical Physics Letters, 79, 347-350. https://doi.org/ 10.1016/0009-2614(81)80219-9

10. Taniguchi, M., Lindsey, J. S., Bocian, D. F., \& Holten, D. (2021). Comprehensive review of photophysical parameters $\left(\varepsilon, \Phi_{f,} \tau_{s}\right)$ of tetraphenylporphyrin $\left(\mathrm{H}_{2} \mathrm{TPP}\right)$ and zinc tetraphenylporphyrin (ZnTPP) - Critical benchmark molecules in photochemistry and photosynthesis. Journal of Photochemistry and Photobiology C: Photochemistry Reviews, 47, 100401. https://doi.org/10.1016/j. jphotochemrev.2020.100401
11. Weil, T., Vosch, T., Hofkens, J., Peneva, K., \& Müllen, K. (2010). The rylene colorant family-tailored nanoemitters for photonics research and applications. Angewandte Chemie International Edition, 49, 9068-9093. https://doi.org/10.1002/anie.200902532

12. Pschirer, N. G., Kohl, C., Nolde, F., Qu, J., \& Müllen, K. (2006). Pentarylene- and hexarylenebis(dicarboximide)s: Near-infraredabsorbing polyaromatic dyes. Angewandte Chemie International Edition, 45, 1401-1404. https://doi.org/10.1002/anie.200502998

13. Hirata, Y., \& Mataga, N. (1993). Electron photoejection and related phenomena in solutions - Ultrafast laser photolysis studies. Progress in Reaction Kinetics, 18, 273-308.

14. Koga, M., Yoneda, Y., Sotome, H., \& Miyasaka, H. (2020). Ionization dynamics of a phenylenediamine derivative in solutions as revealed by femtosecond simultaneous and stepwise two-photon excitation. Physical Chemistry Chemical Physics: PCCP, 21, 2889-2898. https://doi.org/10.1039/C8CP06530F

15. Kawakami, T., Koga, M., Sotome, H., \& Miyasaka, H. (2020). Ultrafast capture of electrons ejected by photoionization leading to the formation of a charge-separated state at a high energy level. Physical Chemistry Chemical Physics: PCCP, 22, 17472-17481. https://doi.org/10.1039/D0CP02029J 